\title{
Similarity Analysis for the Dispersion of Spiraling Modes on Metallic Nanowire to a Planar Thin Metal Layer
}

\author{
Dong-Jin Lee ${ }^{1,2}$, Se-Geun Park ${ }^{1,2}$, Seung-Gol Lee ${ }^{1,2}$, and Beom-Hoan $0^{1,2 *}$ \\ ${ }^{1}$ School of Information and Communication Engineering, Inha University, Incheon 402-751, Korea \\ ${ }^{2}$ LED-Smart Technology Advanced Research (LED-STAR) Center, Inha University, Incheon 402-751, Korea
}

(Received October 14, 2013 : revised November 21, 2013 : accepted November 21, 2013)

\begin{abstract}
We propose a simple model to elucidate the dispersion behavior of spiraling modes on silver nanowire by finding correspondence parameters and building a simple equivalent relationship with the planar insulator-metal-insulator geometry. The characteristics approximated for the proposed structure are compared with the results from an exact solution obtained by solving Maxwell's equation in cylindrical coordinates. The effective refractive index for our proposed equivalent model is in good agreement with that for the exact solution in the $400-2000 \mathrm{~nm}$ wavelength range. In particular, when the radius of the silver nanowire is $100 \mathrm{~nm}$, the calculated index shows typical improvements; the average percentage error for the real part of the effective refractive index is reduced to only $5 \%$ for the $0^{\text {th }}$ order mode $(11.9 \%$ in previous results) and $1.5 \%$ for the $1^{\text {st }}$ order mode $(24.8 \%$ in previous results) in the $400-800 \mathrm{~nm}$ wavelength range. This equivalent model approach is expected to provide further insight into understanding the important behavior of nanowire waveguides.
\end{abstract}

Keywords : Silver nanowire, Planar insulator-metal-insulator geometry

OCIS codes : (240.6680) Surface plasmons; (250.5403) Plasmonics; (230.7370) Waveguides

\section{INTRODUCTION}

In realizing integrated nanophotonic circuits, a promising key is the use of surface plasmons, collective electron oscillations that allow the localization, confinement, and transmission of electromagnetic energy [1, 4]. Various geometries for plasmonic waveguides and integrated components have been suggested; these include grooves and slots in metal films [2-5], metal strips [6-8], dielectric strips deposited on a metal film [9], metal caps on a siliconon-insulator rib waveguide [10-12], and others [13-15].

An alternative approach to achieve integrated nanophotonic circuits involves utilizing the nanowire (NW)-based plasmonic waveguides, which are typical one-dimensional waveguide structures with relatively low losses in visible and nearinfrared spectral ranges [16-20]. To understand the properties of NW-based waveguides, it is essential to investigate their modal dispersion behavior. Schmidt and Russell established a connection between the dispersion of propagating modes on NW geometry and the dispersion of those on planar metal-insulator geometry with a single interface [17]. For an NW radius less than $100 \mathrm{~nm}$, however, there is wide discrepancy owing to interaction with the electromagnetic field.

In this study, we propose a simple model to elucidate the dispersion behavior of spiraling modes on silver nanowire by building a relationship with the planar insulatormetal-insulator geometry. This approximated analysis is compared with a more exact solution obtained by solving Maxwell's equation in cylindrical coordinates. Schmidt and Russel previously established a connection between the dispersion of propagating modes on an NW geometry and those on a planar metal-insulator geometry when the radius of the NW is much larger than the skin depth [17]. For a Ag NW with a radius of $100 \mathrm{~nm}$, the average percentage error for the fundamental mode of the real component of the effective refractive index was reduced from $11.9 \%$ (previous result [17]) to $5 \%$ in the $400-800 \mathrm{~nm}$ wavelength range. The average percentage error for the $1^{\text {st }}$ order mode was reduced from $24.8 \%$ (previous result [17]) to $1.5 \%$ in

\footnotetext{
*Corresponding author: obh@inha.ac.kr

Color versions of one or more of the figures in this paper are available online.
} 
the 400-800 nm wavelength range. This approach provides important insights into the behavior of NW waveguides. Further, it has much broader applicability than previous methods and it can be extended to provide insights into more complicated NW-based waveguides.

\section{METHODS}

An infinitely long and straight Ag NW (with radius $a$ ) was oriented along the $z$-axis and surrounded by silica as illustrated in Fig. 1(a). We found the modal dispersion relation of the Ag NW by building a relationship with the planar insulator-metal-insulator (IMI) geometry as shown in Fig. 1(b). It is worth noting that there has been previous work to investigate the surface plasmon propagation and confinement in IMI geometry [22]. Solving Maxwell's equations using the boundary conditions for the IMI geometry, we obtained the dispersion relation (details are given in the Appendix.),

$$
\varepsilon_{A g} k_{y d, I M I}+\varepsilon_{S O_{2}} k_{y m, I M I} \operatorname{coth}\left(k_{y m, I M I} \frac{D_{v}}{2}\right)=0
$$

where $k_{y m, d}^{2}=\beta_{I M I}^{2}-\varepsilon_{m, d} k_{0}^{2}, k_{0}=\omega / c$ is the free-space wavevector, $\varepsilon_{m}, \varepsilon_{d}$ are the relative permittivity in the $\mathrm{Ag}$ and $\mathrm{SiO}_{2}$, respectively. The dielectric constant for $\mathrm{Ag}$ is taken

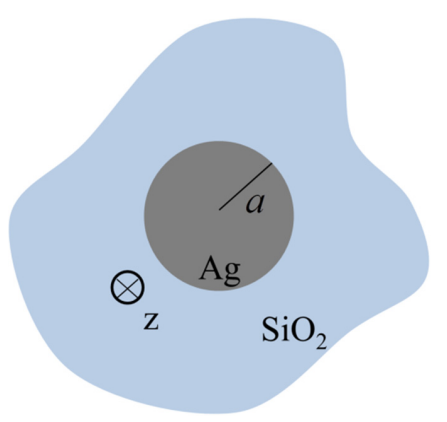

(a)

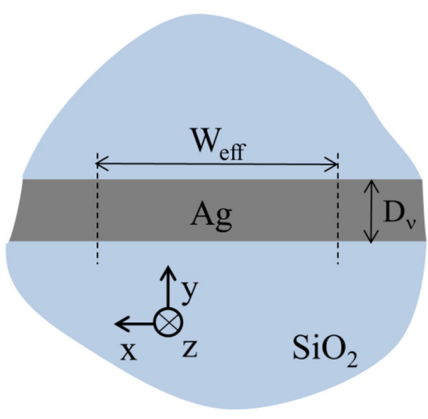

(b)

FIG. 1. (a) An infinitely long and straight Ag NW (radius $a$ ) is oriented along the $z$-axis and surrounded by silica. (b) The proposed equivalent model with planar insulator-metal-insulator (IMI) geometry. from experimental data [21], the real-valued dielectric constant for $\mathrm{SiO}_{2}$ is fixed to $(1.47)^{2}$. The effective thickness of the metal layer $D_{v}$ is expressed by

$$
\mathrm{D}_{v}=\mathrm{D}_{0}+\frac{v}{2} D_{0}
$$

where $v$ is the mode order. The propagating mode in the Ag NW is influenced by the finite cross-sectional area of $\mathrm{NW}$, however, the propagating mode in the IMI geometry is infinitely extended in the $x$-direction. Therefore we needed to define the effective width along to the $\mathrm{x}$-axis to build an equivalent relationship with $\mathrm{Ag} \mathrm{NW}$, and we assumed that the cross-sectional area of the NW was equal to the effective metal area of the IMI geometry, described by

$$
\pi a^{2}=W_{e f f} \times D_{0}
$$

for the fundamental mode. Here, $W_{\text {eff }}$ is defined as a perimeter of an effective mode area for fundamental mode, that is to say $W_{\text {eff }}=2 \pi(a+\delta)$, where $\delta$ represents the effective field radius and defined as the distance required for the electric field intensity to decay by a factor of $1 / e$ along the radial direction of the $\mathrm{Ag} \mathrm{NW}$, as shown in the inset image of Fig. 2. We assumed that the propagating mode had its total wavevector determined by the IMI dispersion relation,

$$
\beta_{I M I}^{2}=\beta^{2}+\left(\frac{v}{a+\delta}\right)^{2},
$$

where $\beta$ is the component of the wavevector along the propagating axis of the $\mathrm{Ag} \mathrm{NW}[15,17]$. Combining Eqs. (1)-(4), we obtain the equivalent dispersion relation for the

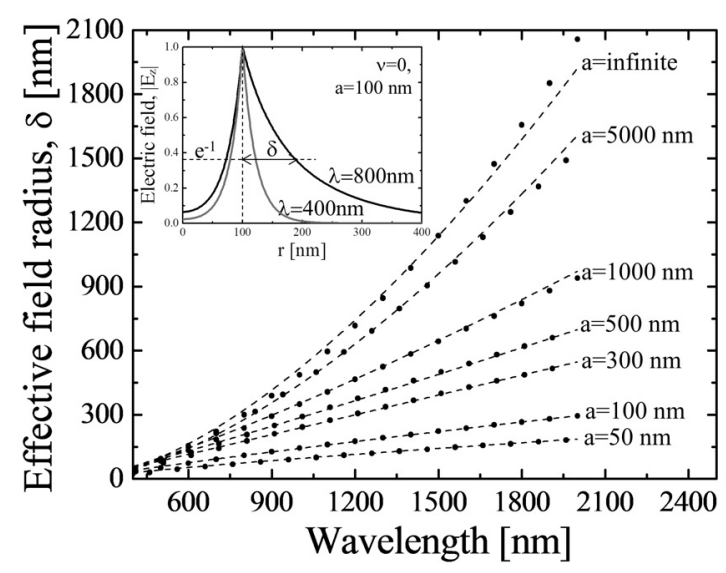

FIG. 2. The effective field radius $\delta$ as a function of the radius and the wavelength. The black dots are obtained from an exact solution (Eq. (6)) and the black dashed lines are obtained from our analytical solution (Eq. (7)). The inset shows the radial dependence of the $z$-components of the electric field for $v=0$ and $a=100 \mathrm{~nm}$ at wavelengths of $400 \mathrm{~nm}$ and $800 \mathrm{~nm}$. 
Ag NW, described by

$$
\begin{array}{r}
\operatorname{coth}\left(\left(\sqrt{\beta^{2}+(v /(a+\delta))^{2}-\varepsilon_{m} k_{0}^{2}}\right) \cdot \frac{\pi a^{2}}{2 \pi(a+\delta)} \cdot\left(1+\frac{v}{2}\right)\right) \\
=-\frac{\varepsilon_{m}\left(\sqrt{\beta^{2}+(v /(a+\delta))^{2}-\varepsilon_{d} k_{0}^{2}}\right)}{\varepsilon_{d}\left(\sqrt{\beta^{2}+(v /(a+\delta))^{2}-\varepsilon_{m} k_{0}^{2}}\right)} .
\end{array}
$$

To obtain the effective field radius $\delta$, we used a direct a direct solution of the dispersion relation of the Ag NW obtained by solving Maxwell's equation in cylindrical coordinates, as follows [23]:

$$
\left[\frac{J_{v}^{\prime}(u)}{u J_{v}(u)}+\frac{K_{v}^{\prime}(w)}{w K_{v}(w)}\right]\left[\frac{J_{v}^{\prime}(u)}{u J_{v}(u)}+\frac{\varepsilon_{d}}{\varepsilon_{m}} \frac{K_{v}^{\prime}(w)}{w K_{v}(w)}\right]=v^{2}\left(\frac{1}{u^{2}}+\frac{1}{w^{2}}\right)\left[\frac{1}{u^{2}}+\frac{\varepsilon_{d}}{\varepsilon_{m}} \frac{1}{w^{2}}\right],
$$

where $J_{v}$ is a Bessel function of the first kind, $J_{v}^{\prime}$ is a derivative of $J_{v}, K_{v}$ is a modified Bessel function of the second kind, $K_{v}^{\prime}$ is a derivative of $K_{v}, u=a \sqrt{k_{0}^{2} \varepsilon_{m}-\beta^{2}}$, $w=a \sqrt{\beta^{2}-k_{0}^{2} \varepsilon_{d}}$.

The propagation constant $\beta$ was calculated by solving Eq. (6). Using these results, we obtained the radial dependence of the z-components of the electric field and the $\delta$ as shown in inset image of Fig. 2. Figure 2 shows the effective field radius $\delta$ as a function of the radius $a$ and the wavelength $\lambda$. The $\delta$ obtained from Eq. (6) is indicated by the black dots. The analytical expression indicated by the black dashed lines was obtained by a fitting of the $\delta$ obtained from Eq. (6) and is described by

$$
\delta(a, \lambda)=A\left(1+B e^{-a / \tau_{a}}\right)\left(\lambda-\lambda_{0}\right)^{f(a)},
$$

where $A=0.01349, B=33.55$, and $\tau_{a}=611.6$. Here, $f(a)=$ $f_{\infty}\left(1-\gamma_{1} e^{-a / a_{01}}-\gamma_{2} e^{-a / a_{02}}\right)$ where $f_{\infty}=1.58587, \gamma_{1}=0.4437, a_{01}$ $=1499.3, \gamma_{2}=0.1271$, and $a_{02}=66.2$.

\section{RESULTS AND DISCUSSION}

The complex values of the effective refractive index $n_{e}=n_{e R}+i n_{e I}$ are yielded from Eq. (5) and (6). Figure 3 shows the effective refractive index of the first three azimuthal mode orders as a function of the wavelength. The top panel shows the real and imaginary parts of the effective refractive index for a radius of $100 \mathrm{~nm}$, and the bottom panel shows the same for a radius of $500 \mathrm{~nm}$. For a radius of $100 \mathrm{~nm}$, the real part of the effective refractive index for the exact solution was in good agreement with that for our approximate value in the $400-1400 \mathrm{~nm}$ wavelength range. Small discrepancies were noticed between the exact and approximate solutions for the imaginary part of the effective refractive index (shown in the inset of the top panel of Fig. 3. For a radius of $500 \mathrm{~nm}$, the real and imaginary parts of the effective refractive index for the exact solution were in good agreement with those for our approximate value in the $400-1400 \mathrm{~nm}$ wavelength range.

We now examine in detail the percentage error of the real part of the effective refractive index and the propagation length in the $400-800 \mathrm{~nm}$ wavelength range as shown in Fig. 4. For the real part of the effective refractive index, the percentage error $\left(n_{e R}\right)$ is defined by $100 \times$ $\left(\left|n_{e R, \text { exact }}-n_{e R, \text { approx. }}\right| / n_{e R, \text { exact }}\right)$, and the approximated model proposed is low-value for the whole wavelength range compared with the previous planar metal-insulator geometry as shown in Fig. 4(a) [17]. The average percentage error for the fundamental mode was reduced to $5 \%$ compared with the previous result of $11.9 \%$, and that for the $1^{\text {st }}$ order mode was reduced to $1.5 \%$ compared with the previous result of $24.8 \%$.

Figure 4(b) shows the percentage error $\left(\mathrm{L}_{\mathrm{P}}\right)$ of the propagation length. The propagation length is given by $L_{P}=1 /\left(2 n_{e l} k_{0}\right)$, and the percentage error $\left(\mathrm{L}_{\mathrm{P}}\right)$ is defined by $100 \times\left(\left|L_{P, \text { exact }}-L_{P, \text { approx. }}\right| / L_{P, \text { exact }}\right)$. The average percentage error for the fundamental mode was reduced to $48 \%$ compared with the previous result of $121.5 \%$, and that for the $1^{\text {st }}$ order mode was slightly increased $39.4 \%$ compared with the previous result of $36.2 \%$.

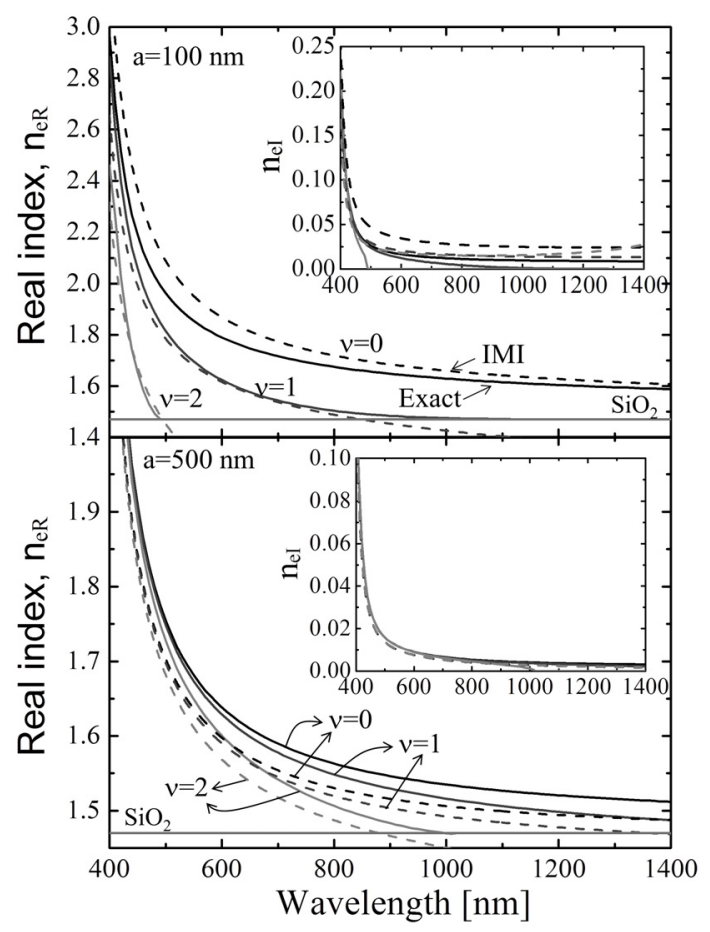

FIG. 3. The effective refractive index of the first three azimuthal mode orders as a function of the wavelength. The real and imaginary parts of the effective refractive index (top panel) for a radius of $100 \mathrm{~nm}$ and (bottom panel) for a radius of $500 \mathrm{~nm}$. The grey solid line corresponds to the bulk material refractive index of $\mathrm{SiO}_{2}$. 


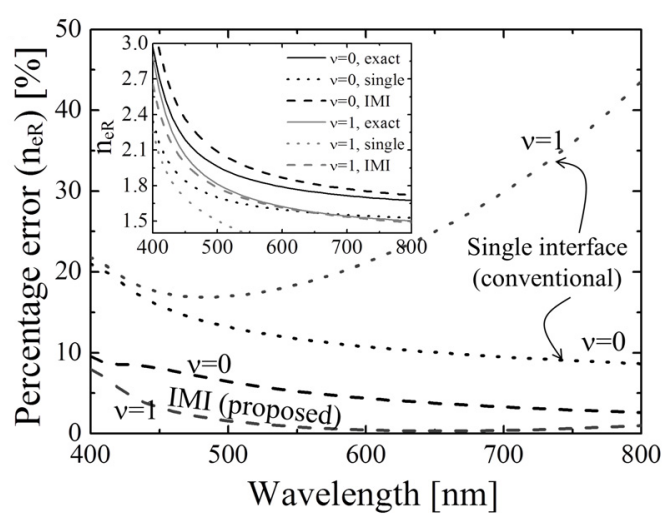

(a)

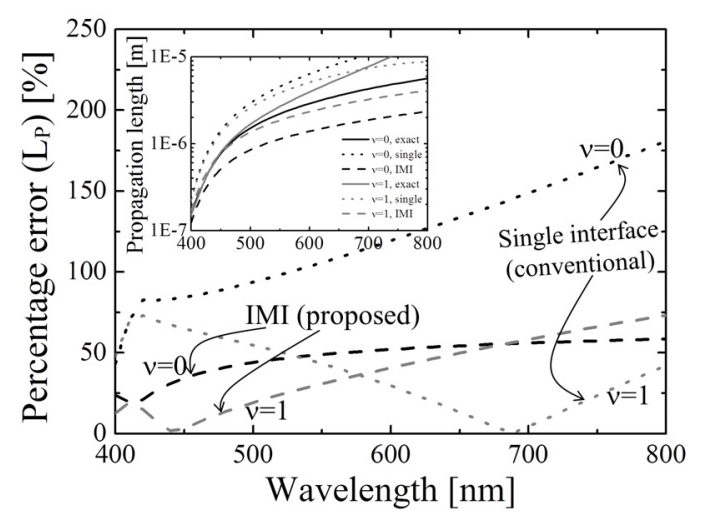

(b)

FIG. 4. (a) The percentage error $\left(n_{e R}\right)$ for the real part of the effective refractive index in the $400-800 \mathrm{~nm}$ wavelength range. The dashed lines and the dotted lines correspond to the proposed IMI geometry and the previous metal-insulator geometry, respectively. Inset shows the effective refractive index as a function of the wavelength from the exact solution (solid lines), previous metal-insulator geometry (dotted lines), and proposed IMI geometry (dashed lines). (b) The percentage error ( $\mathrm{L}_{\mathrm{P}}$ ) of the propagation length in the $400-800 \mathrm{~nm}$ wavelength range. Inset shows the propagation length as a function of the wavelength from the exact solution (solid lines), previous metal-insulator geometry (dotted lines), and proposed IMI geometry (dashed lines).

\section{CONCLUSION}

In summary, we proposed a simple model to elucidate the dispersion behavior of spiraling modes on $\mathrm{Ag} \mathrm{NW}$ by building a relationship with the planar IMI geometry. The previous model with planar metal-insulator geometry has large discrepancies compared with the exact solution when the radius of NW is compatible with the skin depth, due to interactions with the electric field around the NW. Our equivalent model solved this problem by introducing plasmon modes that exist on thin metal films sandwiched between two regions of identical dielectrics, and the percentage error was remarkably reduced. This approach provides important insights into the behavior of NW waveguides. In addition, it has much broader applicability than the previous model and it can be extended to provide insights into more complicated NW-based waveguides.

\section{Appendix. Derivation of Dispersion Relation for IMI Geometry}

For transverse magnetic (TM) polarization (magnetic field along to the x-axis), the electromagnetic fields take the following form:

$$
\begin{aligned}
& \stackrel{\perp}{H}=H_{x} e^{i\left(k_{z} z-\omega t\right)} \hat{x} \\
& \stackrel{r}{E}=E_{y} e^{i\left(k_{z} z-\omega t\right)} \hat{y}+E_{z} e^{i\left(k_{z} z-\omega t\right)} \hat{z} .
\end{aligned}
$$

Inside the metal layer, the field components may be written as:

$$
\begin{aligned}
& E_{z}=e^{k_{y m} y} \pm e^{-k_{y m} y}, \\
& E_{y}=-i \frac{k_{z}}{k_{y m}}\left(e^{k_{y m} y} \mathrm{~m} e^{-k_{y m} y}\right), \\
& H_{x}=\frac{i \omega \varepsilon_{m}}{\mu_{0} c^{2} k_{y m}}\left(e^{k_{y m} y} \mathrm{~m}^{-k_{y m} y}\right) .
\end{aligned}
$$

Outside the metal layer, the field components are given by:

$$
\begin{aligned}
& E_{z}=\left(e^{k_{y m} \cdot D_{v} / 2} \pm e^{-k_{y m} \cdot D_{v} / 2}\right) e^{-k_{y d}\left(y-D_{v} / 2\right)} \\
& E_{y}=i\left(e^{k_{y m} \cdot D_{v} / 2} \pm e^{-k_{y m} \cdot D_{v} / 2}\right) \frac{k_{z}}{k_{y d}} e^{-k_{y d}\left(y-D_{v} / 2\right)} \\
& H_{x}=-i\left(e^{k_{y m} \cdot D_{v} / 2} \pm e^{-k_{y m} \cdot D_{v} / 2}\right) \frac{\omega \varepsilon_{d}}{\mu_{0} c^{2} k_{y d}} e^{-k_{y d}\left(y-D_{v} / 2\right)}
\end{aligned}
$$

where $\mu_{0}$ is the vacuum permeability, $c$ is the speed of light in vacuum, $\varepsilon_{m}, \varepsilon_{d}$ are the relative permittivity in the $\mathrm{Ag}$ and $\mathrm{SiO}_{2}$, respectively. Here, $k_{y m, d}$ is defined by momentum conservation as follows:

$$
k_{y m, d}^{2}=\beta_{I M I}^{2}-\varepsilon_{m, d} k_{0}^{2},
$$

where $k_{0}=\omega / c$ is the free-space wavevector. The IMI waveguides support the two geometry modes - symmetric and antisymmetric - because of the coupling between the two metal-dielectric interfaces in the geometry. We define the mode symmetry in terms of the magnetic field $\left(H_{x}\right)$ with respect to the waveguide median. Solving the Eq. (2)-(4) using the boundary condition, we find the following dispersion relation for antisymmetric mode:

$$
\varepsilon_{m} k_{y d}+\varepsilon_{d} k_{y m} \operatorname{coth}\left(k_{y m} \cdot \frac{D_{v}}{2}\right)=0 .
$$


The effective refractive index of $\mathrm{Ag} \mathrm{NW}$ increases as the radius of NW becomes smaller. On the other hand, the effective refractive index for symmetric mode decreases as the metal layer thickness becomes thinner. Thus, we choose the antisymmetric mode of IMI geometry for equivalent model of Ag NW.

\section{ACKNOWLEDGMENT}

This work was supported in part by Inha university and the MSIP (Ministry of Science, ICT \& Future Planning), Korea, under the C-ITRC (Convergence Information Technology Research Center) support program supervised by the NIPA (National IT Industry Promotion Agency) (NIPA-2013H0301-13-1010).

\section{REFERENCES}

1. H. Raether, Surface Plasmons on Smooth and Rough Surfaces and on Gratings (Springer-Verlag, London, UK, 1998).

2. J. A. Dionne, L. A. Sweatlock, H. A. Atwater, and A. Polman, "Plasmon slot waveguides: Towards chip-scale propagation with subwavelength-scale localization," Phys. Rev. B 73, 035407 (2006).

3. J. Chen, G. A. Smolyakov, S. R. J. Brueck, and K. J. Malloy, "Surface plasmon modes of finite, planar, metalinsulator-metal plasmonic waveguides," Opt. Express 16, 14902-14909 (2008).

4. S. I. Bozhevolnyi, V. S. Volkov, E. Devaux, J.-Y. Laluet, and T. W. Ebbesen, "Channel plasmon subwavelength waveguide components including interferometers and ring resonators," Nature 440, 508-511 (2006).

5. L. Liu, Z. Han, and S. He, "Novel surface plasmon waveguide for high integration," Opt. Express 13, 6645-6650 (2005).

6. P. Berini, R. Charbonneau, N. Lahoud, and G. Mattiussi, "Characterization of long-range surface-plasmon-polariton waveguides,” J. Appl. Phys. 98, 043109 (2005).

7. P. Berini, "Plasmon-polariton waves guided by thin lossy metal films of finite width: Bound modes of asymmetric structures," Phys. Rev. B 63, 125417 (2001).

8. P. Berini, "Plasmon-polariton waves guided by thin lossy metal films of finite width: Bound modes of symmetric structures," Phys. Rev. B 61, 10484-10503 (2000).
9. T. Holmgaard, Z. Chen, S. I. Bozhevolnyi, L. Markey, and A. Dereux, "Dielectric-loaded plasmonic waveguide-ring resonators," Opt. Express 17, 2968-2975 (2009).

10. D. Dai and S. He, "A silicon-based hybrid plasmonic waveguide with a metal cap for a nano-scale light confinement," Opt. Express 17, 16646-16653 (2009).

11. D. Dai and S. He, "Low-loss hybrid plasmonic waveguide with double low-index nano-slots," Opt. Express 18, 1795817966 (2010).

12. Y. Su, Z. Zheng, Y. Bian, L. Liu, X. Zhao, J. Liu, T. Zhou, S. Guo, W. Niu, Y. Liu, and J. Zhu, "Metal-coated hollow nanowires for low-loss transportation of plasmonic modes with nanoscale mode confinement," J. Optics-UK 14, 095501 (2012).

13. A. V. Krasavin and A. V. Zayats, "Silicon-based plasmonic waveguides," Opt. Express 18, 11791-11799 (2010).

14. A. Karalis, E. Lidorikis, M. Ibanescu, J. D. Joannopoulos, and M. Soljacic, "Surface-plasmon-assisted guiding of broadband slow and subwavelength light in air," Phys. Rev. Lett. 95, 063901 (2005).

15. P. B. Catrysse and S. Fan, "Understanding the dispersion of coaxial plasmonic structures through a connection with the planar metal-insulator-metal geometry," Appl. Phys. Lett. 94, 231111 (2009).

16. W. Wang, Q. Yang, F. Fan, H. Xu, and Z. L. Wang, "Light propagation in curved silver nanowire plasmonic waveguides," Nano Lett. 11, 1603-1608 (2011).

17. M. A. Schmidt and P. St. J. Russell, "Long-range spiralling surface plasmon modes on metallic nanowires," Opt. Express 16, 13617-13623 (2008).

18. H. Wei and H. Xu, "Nanowire-based plasmonic waveguides and devices for integrated nanophotonic circuits," Nanophotonics 1, 155-169 (2012).

19. Q. Li and M. Qiu, "Plasmonic wave propagation in silver nanowires: guiding modes or not?" Opt. Express 21, 85878595 (2013).

20. Y. Wang, Y. Ma, X. Guo, and L. Tong, "Single-mode plasmonic waveguiding properties of metal nanowires with dielectric substrates," Opt. Express 20, 19006-19015 (2012).

21. E. D. Palik, Handbook of Optical Constants of Solids (Academic Press, London, UK, 1985).

22. J. A. Dionne, L. A. Sweatlock, H. A. Atwater, and A. Polman, "Planar metal plasmon waveguides: frequency-dependent dispersion, propagation, localization, and loss beyond the free electron model," Phys. Rev. B 72, 075405 (2005).

23. K. Okamoto, Fundamentals of Optical Waveguides (Elsevier Inc., Oxford, UK, 2006). 